\title{
Orbital disproportionation of conduction-electron density in cubic lattices with threefold degenerate site orbitals
}

\author{
L. F. Chibotaru* and A. Ceulemans \\ Department of Chemistry, University of Leuven, Celestijnenlaan 200F, B-3001 Leuven, Belgium
}

(Received 18 December 1995)

\begin{abstract}
The instability of a high-symmetry distribution of conduction electrons in $t$-type orbitals in cubic lattices is investigated within the mean-field approach. All essential bielectronic and one-site vibronic interactions involving the threefold degenerate orbitals are taken into account. It is shown that the degeneracy of the site orbitals may give rise to a special kind of electronic instability, described as orbital disproportionation of the conduction-electron density at each site. The criterion for instability of the cubic band structure with respect to a homogeneous disproportionation, including both spin- and charge-density redistributions, is established. Low-symmetry nuclear distortions may reinforce the tendency for disproportionation of charge density between $t$ orbitals. A model calculation suggests that electronic charge disproportionation may be responsible for the unusual band structure of alkali-metal-doped fullerides. [S0163-1829(96)05423-9]
\end{abstract}

The electronic structure of cubic crystals with partially filled threefold degenerate site orbitals often turns out to be unstable. For example, the cubic perovskite $\mathrm{LaTiO}_{3}$ containing $d^{1}\left(t_{2 g}\right)$ titanium ions is a Mott-Hubbard insulator. ${ }^{1}$ The most interesting examples, however, are offered by the recently discovered ${ }^{2}$ and intensively investigated alkali-metaldoped fullerides. ${ }^{3}$ Each fullerene molecule in the cubic lattice of ordered $A_{x} \mathrm{C}_{60}(A=\mathrm{K}, \mathrm{Rb}, \mathrm{Cs})$ has $T_{h}$ site symmetry, which does not split the degeneracy of the $t_{1 u}$ lowest unoccupied molecular orbitals (LUMO). Many band-structure calculations of $A_{x} \mathrm{C}_{60}$ have been performed, ${ }^{3-9}$ all of them predicting a rigidlike behavior of the $t_{1 u}$ LUMO band irrespective of its population and, therefore, metallic character of the compounds with $0<x<6$. Except for the band-insulator compound $A_{6} \mathrm{C}_{60}$ and pristine $\mathrm{C}_{60}$, where correct bandwidths were obtained, such predictions are in striking contrast with experiment. Thus, the occupied bandwidths of $\mathrm{Rb}_{3} \mathrm{C}_{60}$ and $\mathrm{K}_{3} \mathrm{C}_{60}$ observed in photoemission studies ${ }^{10-13}$ amount to $\sim 1 \mathrm{eV}$, much larger than the calculated total bandwidth of $\sim 0.6 \mathrm{eV}$, and the compounds $A_{4} \mathrm{C}_{60}$ were found to be insulators, ${ }^{12-14}$ which is supported also by resistivity ${ }^{15}$ and electron spin resonance ${ }^{16}$ measurements.

In principle, these discrepancies could be due to structural disorder or many-body effects. It was shown by Hückel-type calculations $^{5-8}$ that merohedral disorder in $A_{3} \mathrm{C}_{60}$ (Ref. 17) can lead to an enlargement of the $t_{1 u}$ LUMO band by up to $20 \%$, which is quite insufficient to reach agreement with experimental data. A comparison of the estimated intraball Coulomb interactions in fullerides ${ }^{18,19}$ with the conduction bandwidth leads to the conclusion that a Mott-Hubbard transition could arise in these materials. ${ }^{12,18,20}$ It was realized, however, in the case of both fullerides ${ }^{18}$ and cubic perovskites such as $\mathrm{SrVO}_{3}$ and $\mathrm{LaTiO}_{3},{ }^{21}$ that such a transition could not be described by a simple Hubbard-type Hamiltonian, due to the orbital degeneracy of the sites. Hence there is clearly a need for a systematic investigation of many-body effects in a band system based on degenerate orbitals. In the present paper we attack this problem with a simplified model. It takes into account all essential bielectronic and one-site vibronic interactions, which involve threefold degenerate site orbitals. The static electronic correlations are considered within the mean-field approximation, using broken-symmetry crystal orbitals. We expect that such an approach, also known as Hartree-Fock instability, ${ }^{22}$ will predict the correct tendency for electronic correlations in this model, as is the case for more simple systems. ${ }^{23}$

The model under investigation is described by the following Hamiltonian containing transfer, bielectronic, and vibronic parts:

$$
\begin{gathered}
\mathcal{H}=\mathcal{H}_{t}+\mathcal{H}_{\mathrm{bi}}+\mathcal{H}_{\mathrm{vib}}, \\
\mathcal{H}_{t}=\sum_{\mathbf{m}, \Delta \mathbf{m}} \sum_{i, j, \sigma} t_{i j}^{\Delta \mathbf{m}^{\prime}} a_{\mathbf{m}, i \sigma}^{\dagger} a_{\mathbf{m}+\Delta \mathbf{m}, j \sigma}, \\
\mathcal{H}_{\mathrm{bi}}=\frac{1}{2} \sum_{\mathbf{m}} \sum_{i, \sigma}\left[U_{\|} n_{\mathbf{m}, i \sigma} n_{\mathbf{m}, i-\sigma}+U_{\perp} \sum_{j \neq i} \sum_{\sigma^{\prime}} n_{\mathbf{m}, i \sigma} n_{\mathbf{m}, j \sigma^{\prime}}\right. \\
-J \sum_{j \neq i}\left(n_{\mathbf{m}, i \sigma} n_{\mathbf{m}, j \sigma}-a_{\mathbf{m}, i \sigma}^{\dagger} a_{\mathbf{m}, j \sigma} a_{\mathbf{m}, i-\sigma}^{\dagger} a_{\mathbf{m}, j-\sigma}\right. \\
\left.-a_{\mathbf{m}, i \sigma}^{\dagger} a_{\mathbf{m}, j \sigma} a_{\mathbf{m}, j-\sigma^{\prime}}^{\dagger} a_{\mathbf{m}, i-\sigma}\right) \\
\left.+\sum_{\Delta \mathbf{m}} U^{\prime}(|\Delta \mathbf{m}|) \sum_{j, \sigma^{\prime}} n_{\mathbf{m}, i \sigma} n_{\mathbf{m}+\Delta \mathbf{m}, j \sigma^{\prime}}\right], \\
\mathcal{H}_{\mathrm{vib}}=\sum_{\mathbf{m}}\left[\frac{1}{2} K_{A} q_{\mathbf{m} a}^{2}+\frac{1}{2} K_{H} \sum_{\gamma} q_{\mathbf{m} \gamma}^{2}+V_{A} \sum_{i, \sigma} n_{\mathbf{m}, i \sigma} q_{\mathbf{m} a}\right. \\
\left.+\sqrt{3 / 5} V_{H} \sum_{i, j, \sigma} \sum_{\gamma} G_{i j}^{\gamma} a_{\mathbf{m}, i \sigma}^{\dagger} a_{\mathbf{m}, j \sigma} q_{\mathbf{m} \gamma}\right],
\end{gathered}
$$

where $i, j=x, y, z$ are the degenerate components of the orbital triplet on each site $\mathbf{m}$. $\mathcal{H}_{t}$ accounts for the transfer-type interactions between sites that are at a distance $\Delta \mathbf{m}$ from each other. $\mathcal{H}_{\mathrm{bi}}$ involves all bielectronic interactions in the $t$ shell on one site. In the case of spherical site symmetry this is described by only two parameters because the relation 
$U_{\|}-U_{\perp}=2 J$ holds. the parameter $U^{\prime}$ determines the repulsion between electrons on different sites. The vibronic part $\mathcal{H}_{\text {vib }}$ includes the interaction with totally symmetric, $\left\{q_{\mathbf{m} a}\right\}$, and nontotally symmetric, $\left\{q_{\mathbf{m} \gamma}\right\}$, one-site nuclear distortions. The latter are treated for simplicity in the limit of degenerate coupling. ${ }^{24}$ Finally $G_{i j}^{\gamma}$ corresponds to a $T_{1} \otimes T_{1}$ Clebsch-Gordan coefficient. ${ }^{25}$

The Hamiltonian in Eq. (1) is averaged over a multielectronic wave function constructed from broken symmetry orbitals of a general form:

$$
\Psi_{g}=\prod_{s, \sigma}^{\mathrm{occ}} a_{s \sigma}^{\dagger}|0\rangle, \quad a_{s \sigma}^{\dagger}=\frac{1}{\sqrt{N}} \sum_{\mathbf{m}, i} c_{\mathbf{m} i}^{s \sigma} a_{\mathbf{m} i \sigma}^{\dagger} .
$$

Here the $c$ coefficients are variational parameters and $N$ is the number of sites. The ground-state energy may be expressed in terms of one-site nuclear distortions and the oneparticle density matrix:

$$
D_{\mathbf{m} i \sigma}^{\mathbf{n} j \sigma}=\frac{1}{N} \sum_{s}^{\mathrm{occ}} c_{\mathbf{m} i}^{s \sigma \star} c_{\mathbf{n} j}^{s \sigma}
$$

In the case of orbitals with cubic symmetry, characterized by momentum $\mathbf{k}$, we have $c_{\mathbf{m} j}^{s \sigma}=e^{i \mathbf{k m}} c_{p \mathbf{k}}^{j}$, where $s$ is now a compound label $(p \mathbf{k})$ with $p=1,2,3$ the index of the band. The real coefficients $\left\{c_{p \mathbf{k}}^{j}\right\}$ for a given $p$ transform as coordinates of a polar vector under the rotations in $\mathbf{k}$ space. This means that the one-site density matrix associated with a cubic band structure is a second-order tensor with nonzero diagonal components $D_{\mathbf{m} i \sigma}^{\mathbf{m} i \sigma} \equiv N_{\mathbf{m} i \sigma}=n / 6$, where $n$ is the total electron population on one site. Hence, a general matrix element in the case of a broken-symmetry band structure can be written as follows:

$$
D_{\mathbf{m} i \sigma}^{\mathbf{m} j \sigma}=\left(n / 6+\Delta N_{\mathbf{m} i \sigma}\right) \delta_{i j}+\Delta D_{\mathbf{m} i \sigma}^{\mathbf{m} j \sigma}\left(1-\delta_{i j}\right),
$$

where $\Delta N_{\mathbf{m} i \sigma}$ and $\Delta D_{\mathbf{m} i \sigma}^{\mathbf{m} j \sigma}(i \neq j)$ now describe the "distortion" of the one-site cubic electron distribution.

The extent of the one-site nuclear distortions is obtained by minimizing the averaged Hamiltonian of Eq. (1) in $q$ space. This yields the following equilibrium values:

$$
\begin{gathered}
q_{\mathbf{m} a}=-\frac{V_{A}}{K_{A}}\left(n+\sum_{i, \sigma} \Delta N_{\mathbf{m} i \sigma}\right), \\
q_{\mathbf{m} \gamma_{1}}=-\sqrt{3 / 5} \frac{V_{H}}{K_{H}} \sum_{i, \sigma} G_{i i}^{\gamma_{1}} \Delta N_{\mathbf{m} i \sigma}, \quad \gamma_{1}=\theta, \epsilon, \\
q_{\mathbf{m} \gamma_{2}}=-\sqrt{3 / 5} \frac{V_{H}}{K_{H i \neq j, \sigma}} \sum_{i j}^{\gamma_{2}} \Delta D_{\mathbf{m} i \sigma}^{\mathbf{m} j \sigma}, \quad \gamma_{2}=\xi, \eta, \zeta,
\end{gathered}
$$

where the manifolds $\gamma_{1}$ and $\gamma_{2}$ belong to irreducible representations $E$ and $T$, respectively, of the cubic site-symmetry group. The summations in Eq. (5) are symmetrized combinations of distortions of the one-site electron distribution. In order to distinguish further the charge $(c)$ and spin $(s)$ disproportionation of the one-site electron density, we introduce variables for sums and differences of spin-up $(\alpha)$ and spindown $(\beta)$ contributions:

$$
\begin{gathered}
\Delta N_{\mathbf{m} i}^{c}=\Delta N_{\mathbf{m} i \alpha}+\Delta N_{\mathbf{m} i \beta}, \quad \Delta N_{\mathbf{m} i}^{s}=\Delta N_{\mathbf{m} i \alpha}-\Delta N_{\mathbf{m} i \beta}, \\
\Delta D_{\mathbf{m} i j}^{c}=\Delta D_{\mathbf{m} i \alpha}^{\mathbf{m} j \alpha}+\Delta D_{\mathbf{m} i \beta}^{\mathbf{m} j \beta}, \quad \Delta D_{\mathbf{m} i j}^{s}=\Delta D_{\mathbf{m} i \alpha}^{\mathbf{m} j \alpha}-\Delta D_{\mathbf{m} i \beta}^{\mathbf{m} j \beta} .
\end{gathered}
$$

Using the Clebsch-Gordan coefficients from Ref. 25 and the variables in Eq. (6), we obtain the following expressions for symmetrized components of the one-site electron distribution: ${ }^{26}$

$$
\begin{gathered}
\Delta N_{\mathbf{m} a}^{c}=1 / \sqrt{3}\left(\Delta N_{\mathbf{m} x}^{c}+\Delta N_{\mathbf{m} y}^{c}+\Delta N_{\mathbf{m} z}^{c}\right), \\
\Delta N_{\mathbf{m} \theta}^{c}=1 / \sqrt{6}\left(-\Delta N_{\mathbf{m} x}^{c}-\Delta N_{\mathbf{m} y}^{c}+2 \Delta N_{\mathbf{m} z}^{c}\right), \\
\Delta N_{\mathbf{m} \epsilon}^{c}=1 / \sqrt{2}\left(\Delta N_{\mathbf{m} x}^{c}-\Delta N_{\mathbf{m} y}^{c}\right), \\
\Delta D_{\mathbf{m} \xi}^{c}=1 / \sqrt{2}\left(\Delta D_{\mathbf{m} y z}^{c}+\Delta D_{\mathbf{m} z y}^{c}\right), \\
\Delta D_{\mathbf{m} \eta}^{c}=1 / \sqrt{2}\left(\Delta D_{\mathbf{m} x z}^{c}+\Delta D_{\mathbf{m} z x}^{c}\right), \\
\Delta D_{\mathbf{m} \zeta}^{c}=1 / \sqrt{2}\left(\Delta D_{\mathbf{m} x y}^{c}+\Delta D_{\mathbf{m} y x}^{c}\right),
\end{gathered}
$$

and similarly for $\Delta N_{\mathbf{m} \gamma_{1}}^{s} \quad\left(\gamma_{1}=a, \theta, \epsilon\right)$ and $\Delta D_{\mathbf{m} \gamma_{2}}^{s}$ $\left(\gamma_{2}=\xi, \eta, \zeta\right)$. By analogy with nuclear distortions the $\Delta N_{\mathbf{m} \gamma_{1}}^{c}$ in Eq. (7) may be said to describe totally symmetric $(a)$, tetragonal $(\theta)$, and orthorhombic $(\epsilon)$ charge disproportionations, while the $\Delta D_{\mathbf{m} \gamma_{2}}^{c}$ refer to monoclinic charge disproportionations on a given site. The same is true for the components of the one-site spin-density redistributions, $\Delta N_{\mathbf{m} \gamma_{1}}^{s}$ and $\Delta D_{\mathbf{m} \gamma_{2}}^{s}$. Substituting back the equilibrium coordinates of Eq. (5) into the averaged Hamiltonian of Eq. (1) and using the symmetrized variables defined in Eq. (7) we obtain the following expressions for bielectronic and vibronic contributions to the ground-state energy:

$$
\left\langle\mathcal{H}_{\mathrm{bi}}+\mathcal{H}_{\mathrm{vib}}\right\rangle=\left\langle\mathcal{H}_{\mathrm{bi}}+\mathcal{H}_{\mathrm{vib}}\right\rangle_{0}+\Delta\left\langle\mathcal{H}_{\mathrm{bi}}+\mathcal{H}_{\mathrm{vib}}\right\rangle
$$

Here the zeroth-order term corresponds to the undistorted, cubic band structure:

$$
\begin{aligned}
\left\langle\mathcal{H}_{\mathrm{bi}}+\mathcal{H}_{\mathrm{vib}}\right\rangle_{0}= & N\left\{\left[\frac{1}{12}\left(U_{\|}+4 U_{\perp}-2 J\right)-\frac{1}{2} \varphi_{M}-E_{A}\right] n^{2}\right. \\
& \left.-\frac{1}{2} \sum_{\Delta \mathbf{m}} U^{\prime}(|\Delta \mathbf{m}|) \sum_{i, j, \sigma}\left|D_{i \sigma}^{j \sigma}(\Delta \mathbf{m})\right|^{2}\right\} .
\end{aligned}
$$

In Eq. (9) $\varphi_{M}$ is the Madelung potential on each site arising from the final term of $\mathcal{H}_{\mathrm{bi}}$, as well as from electrostatic interaction with other ions not included in Eq. (1). The quantity $E_{A}$ represents the one-site energy gain due to relaxation along the totally symmetric nuclear coordinate: $E_{A}=(1 / 2) V_{A}^{2} / K_{A}$. Finally the term in $U^{\prime}$ refers to the Hartree-Fock screening of intercenter Coulomb interaction. The accompanying density matrix element $D_{i \sigma}^{j \sigma}(\Delta \mathbf{m})$ corresponds to the cubic symmetry value of $D_{\mathbf{m}, i \sigma}^{\mathbf{m}+\Delta \mathbf{m}, j \sigma}$. As the notation emphasizes, this undistorted value only depends on the difference $\Delta \mathbf{m}$ between the sites. The cubic bandstructure distortion can be presented in the form 


$$
\begin{aligned}
\Delta\left\langle\mathcal{H}_{\mathrm{bi}}+\mathcal{H}_{\mathrm{vib}}\right\rangle= & \frac{1}{2} \sum_{\mathbf{m}}\left\{K_{a}^{c}\left(\Delta N_{\mathbf{m} a}^{c}\right)^{2}+K_{a}^{s}\left(\Delta N_{\mathbf{m} a}^{s}\right)^{2}\right. \\
& +\sum_{\gamma_{1}=\theta, \epsilon}\left[K_{1}^{c}\left(\Delta N_{\mathbf{m} \gamma_{1}}^{c}\right)^{2}+K_{1}^{s}\left(\Delta N_{\mathbf{m} \gamma_{1}}^{s}\right)^{2}\right] \\
& \left.+\sum_{\gamma_{2}=\xi, \eta, \zeta}\left[K_{2}^{c}\left(\Delta D_{\mathbf{m} \gamma_{2}}^{c}\right)^{2}+K_{2}^{s}\left(\Delta D_{\mathbf{m} \gamma_{2}}^{s}\right)^{2}\right]\right\},
\end{aligned}
$$

with the following coefficients:

$$
\begin{gathered}
K_{a}^{c}=2 U_{\perp}+\frac{1}{2} U_{\|}-J-3 A_{M} U^{\prime}-6 E_{A}, \\
K_{a}^{s}=-\frac{1}{2}\left(U_{\|}+2 J\right), \\
K_{1}^{c}=-\frac{1}{2}\left(2 U_{\perp}-U_{\|}-J+6 E_{H}\right), \\
K_{1}^{s}=-\frac{1}{2}\left(U_{\|}-J\right), \\
K_{2}^{c}=-\frac{1}{2}\left(U_{\perp}-3 J+6 E_{H}\right), \\
K_{2}^{s}=-\frac{1}{2}\left(U_{\perp}+J\right) .
\end{gathered}
$$

Here $A_{M}$ is a Madelung constant and $U^{\prime}$ is shortened notation for electrostatic interactions between nearest-neighbor sites; $E_{H}$ is the stabilization energy after nontotally symmetric vibrations: $E_{H}=(1 / 5) V_{H}^{2} / K_{H}$. In the case of multimode vibronic coupling the $E_{A}$ and $E_{H}$ quantities are obtained by summing the stabilization energies of the participating vibrational modes. ${ }^{27}$ Note that the expression in Eq. (10) is bilinear in the distortion variables of Eq. (7). This is of course due to charge conservation, $\Sigma_{\mathbf{m}} \Delta N_{\mathbf{m} a}=0$, in combination with overall cubic symmetry. In principle we should also have considered, by analogy with the last term of Eq. (9), a contribution from the variation of screening of intercenter Coulomb interaction with the distortion of cubic band structure. This effect is more intricate though since it involves the variation of off-diagonal density elements, connecting different sites. Except for this last contribution, the results in Eqs. (10) and (11) are essentially complete.

Negative values for the curvature constants $K_{i}^{c, s}$ in Eq. (10) will give rise to instability of the high-symmetry ground-state band structure. As can be seen from Eq. (11) all $K$ parameters are indeed expected to be negative, except for $K_{a}^{c}$, which is more likely to be positive. The $K_{a}^{c}$ constant describes the variation of interaction energy under total charge fluctuation on the sites. When a regular lattice of such fluctuations arises, the energy of electrostatic interaction of these new charges, $\Delta N_{\mathbf{m} a}^{c}= \pm \Delta N_{a}^{c}$, related to one site, is described by the fourth term in the expression for $K_{a}^{c}$. The second curvature constant, $K_{a}^{s}$, describes the effect of total spin-density polarization of the sites that can be detected, for instance, as spin-density waves. Both $K_{a}^{c}$ and $K_{a}^{s}$ contributions are isotropic and already arise in the case of nondegenerate sites, i.e., in the Hubbard model. The other parameters in Eq. (11) describe the energetic effect of orbital disproportionation of charge and spin density on each site. This effect can be understood from the following simple consideration. The intrasite electrostatic energy includes the interaction of electrons from each spin orbital with electrons from the five remaining spin orbitals. Because there can be no interelectric interaction within one spin orbital, it is more favorable, for total occupation number $n=1$, to occupy a single spin orbital by one electron than all six spin orbitals by one-sixth of an electron. Note that the energetic effect of such disproportionation cannot be described correctly within density-functional methods unless the self-interaction corrections are taken into account. $^{28}$

It can be expected that the relationship between the constants in Eq. (11) determines in a qualitative manner the leading tendency for electronic correlations. In the case of cubic perovskites $\mathrm{SrVO}_{3}, \mathrm{LaTiO}_{3}, \mathrm{YTiO}_{3}$, etc., the interelectronic interactions in the $d$ shell of titanium and vanadium ions are much stronger than $E_{H}$, a typical value for the latter being $0.1 \mathrm{eV}$. So, we can expect the following relation: $\left|K_{a}^{s}\right|>\left|K_{1,2}^{s}\right|>\left|K_{1,2}^{c}\right|$, which means that spin correlations are the dominant features in these systems. In the case of alkalimetal-doped fullerides the contribution of $6 E_{H}$ is no longer negligible, especially because the intrasite bielectronic parameters are one order of magnitude smaller as compared with cubic perovskites. Using reasonable estimates for these parameters, $U_{\|}=0.8-1.3 \mathrm{eV},{ }^{19} J=50 \mathrm{meV},{ }^{18} U_{\perp}=U_{\|}-2 J$, and $E_{H}=0.1 \mathrm{eV},{ }^{29}$ one obtains the following values in $\mathrm{eV}$ : $K_{1}^{c}=K_{2}^{c}=-0.7, K_{a}^{s}=-0.55$, and $K_{1}^{s}=K_{2}^{s}=-0.5$. The constant $K_{a}^{c}$ is determined by additional intramolecular parameters, $U^{\prime}$ and $E_{A}$, the estimations for which are $U^{\prime}=0.3-0.5 \mathrm{eV}$ (Ref. 19) and $E_{A}=20 \mathrm{meV}$ (Ref. 30). With a normal Madelung constant, $A_{M}=1.5-2.0,{ }^{31} K_{a}^{c}$ is contained in the interval between -0.3 and $0.3 \mathrm{eV}$. It follows from a comparison of these estimates that orbital charge disproportionation of the conduction electrons on each site may be a characteristic feature of fulleride materials.

So far we have been able to show that interelectronic repulsion interaction may provide a driving force for orbital disproportionation. Whether or not this effect will materialize depends on the response of the kinetic part of the groundstate energy, $\left\langle\mathcal{H}_{t}\right\rangle$, under the distortion of the cubic band structure. To obtain a criterion for instability it is enough to consider only small changes of the cubic band structure constructed from orbitals, which are solutions of the "cubic" Fock equations:

$$
\sum_{j}\left[F_{i j}(0)+\sum_{\Delta \mathbf{m}} F_{i j}(\Delta \mathbf{m}) \cos (\mathbf{k} \cdot \Delta \mathbf{m})\right] c_{p \mathbf{k}}^{j}=\varepsilon_{p \mathbf{k}} c_{p \mathbf{k}}^{j},
$$

where the Fock matrix elements are as follows:

$$
\begin{gathered}
F_{i j}(0)=\delta_{i j}\left(U_{\|}+4 U_{\perp}-2 J\right) \frac{n}{6}, \\
F_{i j}(\Delta \mathbf{m})=t_{i j}^{\Delta \mathbf{m}}-U^{\prime}(|\Delta \mathbf{m}|) D_{i \sigma}^{j \sigma}(\Delta \mathbf{m}) .
\end{gathered}
$$

We note in passing that the last term in the expression for $F_{i j}(\Delta \mathbf{m})$ in Eq. (13), not included in Hückel-type calculations, contributes to non-rigid-like behavior of the bands. Its maximal value is achieved for half filling, i.e., for $n=3$. 
Further calculations depend on the type of distortion. Since our primary concern is to obtain a simple model description of the effect of orbital disproportionation, the distortion was assumed to be homogeneous. This means that translational invariance is preserved, although the resulting band structure need no longer be cubic. Accordingly $\Delta N_{\mathbf{m} a}^{c}=0$ while the other variables, $\Delta N_{\mathbf{m} \gamma_{1}}^{c}, \Delta N_{\mathbf{m} \gamma_{1}}^{s}$, $\Delta D_{\mathbf{m} \gamma_{2}}^{c}, \Delta D_{\mathbf{m} \gamma_{2}}^{s}$, and $\Delta N_{\mathbf{m} a}^{s}$, are independent of the site index. The variation of the kinetic (band) energy reflects both the variation of the orbital eigenvectors, $c_{p \mathbf{k}}^{i}+\Delta c_{p \mathbf{k}}^{i}$, and the repopulation of the band states near the Fermi surfaces. The latter results from changes of these surfaces, described by vectors $\Delta \mathbf{k}_{p}\left(\mathbf{k}_{F}^{p}\right)$, perpendicular to a given Fermi surface $(p)$ in each point $\mathbf{k}_{F}^{p}$. The average energy, $\left\langle\mathcal{H}_{t}\right\rangle$, is minimized after $\left\{\Delta c_{p \mathbf{k}}^{i}\right\}$ and $\left\{\Delta \mathbf{k}_{p}\right\}$ under twelve conditions, imposed in order to obtain new orbitals and Fermi surfaces consistent with the twelve parameters of band-structure distortion. Solving the corresponding extrema equations one obtains $\Delta c_{p \mathbf{k}}^{i}$ and $\Delta \mathbf{k}_{p}$ as functions of band-structure distortion parameters. In the lowest order of the perturbation theory, i.e., for small distortions of the cubic band structure, we have linear dependencies on these parameters with the coefficients determined by the following quantities:

$$
\begin{gathered}
A=\frac{1}{(2 \pi)^{3}} \sum_{p} \int_{S_{p}} \frac{\left(c_{p \mathbf{k}}^{i}\right)^{4}}{\left|\nabla \varepsilon_{p \mathbf{k}}\right|} d S_{p}\left(\mathbf{k}_{F}^{p}\right), \\
B=\frac{1}{(2 \pi)^{3}} \sum_{p} \int_{S_{p}} \frac{\left(c_{p \mathbf{k}}^{i}\right)^{2}\left(c_{p \mathbf{k}}^{j}\right)^{2}}{\left|\nabla \varepsilon_{p \mathbf{k}}\right|} d S_{p}\left(\mathbf{k}_{F}^{p}\right), \quad i \neq j, \\
C_{i j, l m}=\frac{1}{(2 \pi)^{3}} \sum_{p_{1}, p_{2}} \int n_{p_{1} \mathbf{k}} \frac{c_{p_{1} \mathbf{k}}^{i} c_{p_{1} \mathbf{k}}^{j} c_{p_{2} \mathbf{k}}^{l} c_{p_{2} \mathbf{k}}^{m}}{\varepsilon_{p_{2} \mathbf{k}}-\varepsilon_{p_{1} \mathbf{k}}} d \mathbf{k},
\end{gathered}
$$

where $S_{p}$ is the Fermi surface and $n_{p \mathbf{k}}=0,1$ is the occupation number of the corresponding band spin orbital for the cubic band structure. The parameters $A$ and $B$ describe the effect of orbital repopulation near the Fermi surfaces. The $C_{i j, l m}$ are components of a fourth-rank tensor describing changes of orbital composition. In a cubic site-symmetry group only three $C$ components are nonzero: $C_{i i, i i}, C_{i i, j j}$, and $C_{i j, i j}$. Because of orthogonality of $c_{p_{1} \mathbf{k}}$ and $c_{p_{2} \mathbf{k}}$ vectors, only two components are independent, i.e., $C_{i i, i i}+2 C_{i j, i j}=0$.

Substituting the obtained solutions for variational parameters $\left\{\Delta c_{p \mathbf{k}}^{i}\right\}$ and $\left\{\Delta \mathbf{k}_{p}\right\}$ into $\left\langle\mathcal{H}_{t}\right\rangle$ we finally obtain an expression for the kinetic part of the energy as a sum of the zeroth-order band energy and a distortion term, $\Delta\left\langle\mathcal{H}_{t}\right\rangle$, which has the same form as Eq. (10) with curvature coefficients:

$$
\begin{gathered}
\widetilde{K}_{a}^{s}=\frac{1}{2}[A+2 B]^{-1}, \\
\widetilde{K}_{1}^{c}=\widetilde{K}_{1}^{s}=\frac{1}{2}\left[A-B+3 C_{11,11}\right]^{-1}, \\
\widetilde{K}_{2}^{c}=\widetilde{K}_{2}^{s}=\frac{1}{2}\left[2 B-C_{11,11}+2 C_{11,22}\right]^{-1} .
\end{gathered}
$$

As follows from their definition, the parameters involved in Eq. (15) are all positive and $A>B$. They depend strongly on the site population number $n$ and are inversely proportional to the bandwidth $w$. They can also be related to empirical quantities. Indeed, we can see that

$$
A+2 B=\rho_{F}^{(i)}=\frac{1}{3} \rho_{F},
$$

where $\rho_{F}^{(i)}$ is the projected density of states corresponding to one orbital $i$, and $\rho_{F}$ is the total density of states per site and per spin at the Fermi level. The analogous combination of the $C$ parameters yields

$$
C_{11,11}+2 C_{11,22}=\frac{1}{6} \sum_{p_{1}, p_{2}} \frac{1}{(2 \pi)^{3}} \int \frac{n_{p_{1} \mathbf{k}}-n_{p_{2} \mathbf{k}}}{\varepsilon_{p_{2} \mathbf{k}}-\varepsilon_{p_{1} \mathbf{k}}} d \mathbf{k},
$$

which has the form of a susceptibility.

It should be pointed out that the obtained orbitals and Fermi surfaces in the above calculations do not correspond to self-consistent Hartree-Fock solutions. This is obvious because such solutions correspond to the minimum of electronic energy within a one-electron approximation, which cannot be achieved for arbitrary values of the parameters in Eq. (7). The true Hartree-Fock band orbitals and Fermi surfaces of the distorted band structure can be obtained only by additional minimization of $\Delta\langle\mathcal{H}\rangle$ after the parameters of band-structure distortion.

A combination of the potential [Eq. (11)] and kinetic [Eq. (15)] contributions to the ground-state energy variation as a function of disproportionation leads to the conclusion that instability arises if (at least) one of the following conditions holds:

$$
K_{i}^{c}+\widetilde{K}_{i}^{c}<0, \quad K_{i}^{s}+\widetilde{K}_{i}^{s}<0 \quad\left(i=a, \gamma_{1}, \gamma_{2}\right)
$$

In the case of a simple cubic lattice the $\widetilde{K}$ constants in this expression can be estimated easily. Indeed in this case the nearest-neighbor hopping does not lead to the hybridization of site orbitals, so we have three equivalent bands corresponding to three orbitals, $c_{p \mathbf{k}}^{i}=\delta_{i p}$, and, as a consequence, $B=C_{11,11}=0$. This implies that the band-structure distortions $\Delta N_{\mathbf{m} \gamma_{1}}^{c}$ and $\Delta N_{\mathbf{m} \gamma_{1}}^{s}$ can only be due to a repopulation of band states near the Fermi surface. Approximating the projected density of states as $\rho_{F}^{i} \approx 1 / w$, we obtain $\widetilde{K}_{a}^{s}=\widetilde{K}_{1}^{c}=\widetilde{K}_{1}^{s}=1 / 2 \rho_{F}^{i} \approx w / 2$, where $w$ is the width of each band. As far as the distortions $\Delta D_{\mathbf{m} \gamma_{2}}^{c}$ and $\Delta D_{\mathbf{m} \gamma_{2}}^{s}$ are concerned, they will be connected only with modification of band orbitals. The expression for the corresponding constants becomes $\widetilde{K}_{2}^{c}=\widetilde{K}_{2}^{s}=1 / C_{11,22}$, which can be obtained by means of the susceptibility term in Eq. (17). In the absence of vibronic contributions the potential constants of Eq. (11), except for $K_{a}^{c}$, are of the order $-U / 2$. Thus, the criterion for instability after $\Delta N_{\mathbf{m a}}^{s}, \Delta N_{\mathbf{m} \gamma_{1}}^{c}$, and $\Delta N_{\mathbf{m} \gamma_{1}}^{s}$ can be written as $w<U$, which is in qualitative agreement with the criterion for the Mott-Hubbard transition. ${ }^{32}$ In the case of fullerides such a relation probably underestimates the extent of instability because the density of states will be higher than the supposed $\rho_{F}=3 / w$, while vibronic terms contained in Eq. (11) also will become important. On the other hand, a hitherto neglected change of the Hartree-Fock screening of intercenter Coulomb interaction upon band-structure distortion tends to increase the $\widetilde{K}$ constants in Eq. (15). Indeed, the distortion of the cubic band structure enhances the localiza- 
TABLE I. Solutions for the distorted band structure of a simple cubic model with integer numbers of electrons per site. In the case of undistorted, cubic band structure, we have $n / 3, n w / 6$, and $w$ for band occupation numbers, occupied bandwidth, and total bandwidth, correspondingly.

\begin{tabular}{|c|c|c|c|c|c|c|c|c|c|}
\hline \multirow[b]{2}{*}{$n$} & \multicolumn{2}{|c|}{$\begin{array}{c}\text { Band-structure } \\
\text { distortion parameters }\end{array}$} & \multicolumn{3}{|c|}{$\begin{array}{l}\text { Band occupation } \\
\text { numbers }\end{array}$} & \multicolumn{3}{|c|}{ Bandwidth } & \multirow{2}{*}{$\begin{array}{c}\text { Correlation } \\
\text { energy per } \\
\text { site }^{\mathrm{a}}\end{array}$} \\
\hline & $\Delta N_{\mathbf{m} \theta}^{c}$ & $\Delta N_{\mathbf{m} \epsilon}^{c}$ & $N_{x}$ & $N_{y}$ & $N_{z}$ & Occupied & Total & Insulating gap & \\
\hline \multirow[t]{2}{*}{1} & $-1 / \sqrt{6}$ & 0 & $\frac{1}{2}$ & $\frac{1}{2}$ & 0 & $w / 4$ & $w-\frac{1}{2} K_{1}^{c}$ & & $1 / 6$ \\
\hline & $2 / \sqrt{6}$ & 0 & 0 & 0 & 1 & $w / 2$ & $w-K_{1}^{c}$ & & $2 / 3$ \\
\hline \multirow[t]{2}{*}{2} & $-2 / \sqrt{6}$ & 0 & 1 & 1 & 0 & $w / 2$ & $w-K_{1}^{c}$ & & $2 / 3$ \\
\hline & $4 / \sqrt{6}$ & 0 & 0 & 0 & 2 & $w$ & $w-2 K_{1}^{c}$ & $-2 K_{1}^{c}-w$ & $8 / 3$ \\
\hline \multirow[t]{4}{*}{3} & $-3 / \sqrt{6}$ & 0 & $\frac{3}{2}$ & $\frac{3}{2}$ & 0 & $\frac{3}{4} w$ & $w-\frac{3}{2} K_{1}^{c}$ & & $3 / 2$ \\
\hline & $3 / \sqrt{6}$ & 0 & $\frac{1}{2}$ & $\frac{1}{2}$ & 2 & $w / 4-\frac{3}{2} K_{1}^{c}$ & $w-\frac{3}{2} K_{1}^{c}$ & & $3 / 2$ \\
\hline & 0 & $\sqrt{2}$ & 2 & 0 & 1 & $w / 2-K_{1}^{c}$ & $w-2 K_{1}^{c}$ & & 2 \\
\hline & 0 & $-\sqrt{2}$ & 0 & 2 & 1 & $w / 2-K_{1}^{c}$ & $w-2 K_{1}^{c}$ & & 2 \\
\hline \multirow[t]{2}{*}{4} & $2 / \sqrt{6}$ & 0 & 1 & 1 & 2 & $w / 2-K_{1}^{c}$ & $w-K_{1}^{c}$ & & $2 / 3$ \\
\hline & $-4 \sqrt{6}$ & 0 & 2 & 2 & 0 & $w$ & $w-2 K_{1}^{c}$ & $-2 K_{1}^{c}-w$ & $8 / 3$ \\
\hline \multirow[t]{2}{*}{5} & $1 / \sqrt{6}$ & 0 & $\frac{3}{2}$ & $\frac{3}{2}$ & 2 & $\frac{3}{4} w-\frac{1}{2} K_{1}^{c}$ & $w-\frac{1}{2} K_{1}^{c}$ & & $1 / 6$ \\
\hline & $-2 / \sqrt{6}$ & 0 & 2 & 2 & 1 & $w / 2-K_{1}^{c}$ & $w-K_{1}^{c}$ & & $2 / 3$ \\
\hline
\end{tabular}

${ }^{\text {an }}$ units of $\frac{1}{2}\left(w / 2+K_{1}^{c}\right)$.

tion of $t_{1}$ electrons, which leads to the diminution of intercenter one-electron matrix elements and, as a consequence, to the decrease of the screening mentioned above. This effect seems to be not negligible in fullerides because $U^{\prime}$ is of the same order as $U_{\|}, U_{\perp}$.

The considered homogeneous distortion of the cubic band structure is not generally expected to be more favorable for the band energy than other types of ordering of orbital density disproportionation at different sites. In the case of fullerides there is an additional effect of orientational disordering of fullerene molecules, which can increase this disproportionation. Indeed, it was shown by calculations ${ }^{5-8}$ that the band energy in $A_{3} \mathrm{C}_{60}$ becomes even lower for merohedrally disordered phases. In these phases the cubic sitesymmetry of the electron transfer operator $\left\langle\mathcal{H}_{t}\right\rangle$ is removed, resulting in orbital disproportionation of one-site charge density already for noninteracting $t_{1 u}$ electrons. The intraball electron repulsion and vibronic interactions will enhance this disproportionation, contributing in turn to additional stabilization of disordered phases in conformity with Eq. (10). The same arguments also seem to be valid for other fulleride stoichiometries, such as $A \mathrm{C}_{60}$ and $A_{4} \mathrm{C}_{60}$, where orientational (not merohedral) disorder of the fullerene molecules was found. ${ }^{33}$ However, to our knowledge, band-structure calculations of $A_{n} \mathrm{C}_{60}$ in disordered phases, taking into account the effects of intraball electronic interactions and the relaxation along $H_{g}$ vibrations, were not performed until now.

The orbital disproportionation of one-site charge density leads to a widening of the whole band, induced by the change of the Fock operator in Eq. (13). The change of the one-site part of this operator, $\Delta F^{\mathbf{m}}(0)$, describes the band splitting in the $\Gamma$ point of the Brillouin zone and has the following form, irrespective of spin:

$$
\Delta F_{i j}^{\mathbf{m}}(0)=K_{1}^{c} \Delta N_{\mathbf{m} i}^{c} \delta_{i j}+K_{2}^{c} D_{\mathbf{m} i j}^{c}\left(1-\delta_{i j}\right) .
$$

The enlargement of the whole band is determined mainly by the differences between eigenvalues of the matrix $\Delta F^{\mathbf{m}}(0)$. When these differences become larger than the total bandwidth, the overlap between some of the three bands disappears and the system can become an insulator for even occupation numbers $n=2,4$.

In order to illustrate this situation consider the instability of a simple cubic lattice with respect to homogeneous tetragonal and orthorhombic one-site charge disproportionation only. Further, assume for simplicity a constant density of states in each band $p, \rho^{(p)}=1 / w(p=x, y, z)$. The variation of the total electronic energy has the form

$$
\Delta\langle\mathcal{H}\rangle=\frac{1}{2}\left(w / 2+K_{1}^{c}\right)\left[\left(\Delta N_{\mathbf{m} \theta}^{c}\right)^{2}+\left(\Delta N_{\mathbf{m} \epsilon}^{c}\right)^{2}\right]
$$




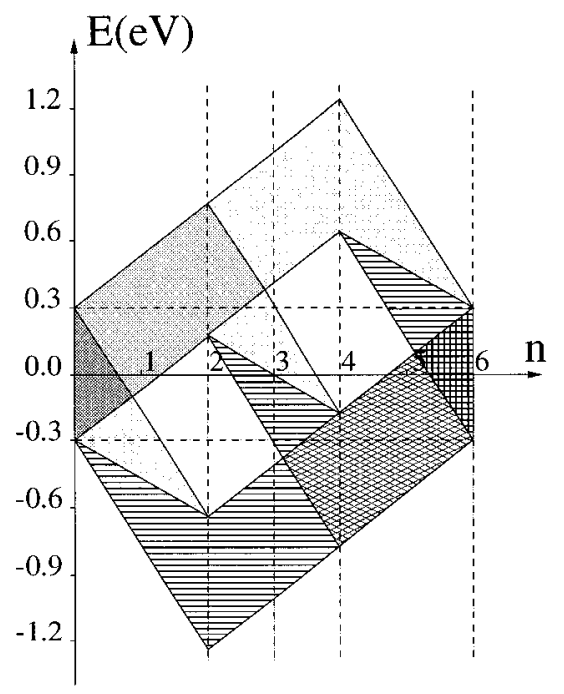

FIG. 1. Ground-state distorted band structure of a simple cubic model as a function of the population of $t_{1 u}$ site orbitals $(n)$. The dashed and dotted fields correspond to occupied and unoccupied band states, respectively. The horizontal lines delineate a rigid band-structure correlation between the fully empty and fully occupied limits. In these limits the band system consists of three overlapping components. As the intensity of the shading indicates, these components are gradually resolved when approaching the $n=3$ occupation in the middle.

which is valid for all allowed (not only small) values of $\Delta N_{\mathbf{m} \theta}^{c}$ and $\Delta N_{\mathbf{m} \epsilon}^{c}$ because of the supposed constant density of states. As established above, in this simple cubic model the parameters $\Delta N_{\mathbf{m} \theta}^{c}$ and $\Delta N_{\mathbf{m} \epsilon}^{c}$ are related only to repopulation of the bands. So the expected distortion of the band structure results merely in repopulation and shifts of the three bands when the criterion for instability, $w / 2+K_{1}^{c}<0$, is fulfilled. The minimization of $\Delta\langle\mathcal{H}\rangle$ after variables $\Delta N_{\mathbf{m} \theta}^{c}$ and $\Delta N_{\mathbf{m} \epsilon}^{c}$, taking into account the allowed region of their values for different $n$ 's, generates several selfconsistent Hartree-Fock solutions for distorted band structure. These are listed in Table I for integer values of $n$. As expected from the form of Eq. (20), the lowest-energy solution is achieved under maximal charge disproportionation for each $n$. These solutions always correspond to an insulating state for $n=2,4$. The energy gain (correlation energy) related to one electron (or hold for $n>3$ ) is $2 / 3$ for $n=1,3,5$ and $4 / 3$ for $n=2,4$ in units of $\frac{1}{2}\left(w / 2+K_{1}^{c}\right)$. The gain by charge disproportionation is thus larger for $n=2,4$ than for $n=1,3,5$. The latter have a half-filled upper band $(p=z$ in Table I) and, therefore, can be subjected to further instability involving spin-density disproportionation. The evolution of the band structure as a continuous function of $n$ is shown in Fig. 1 for parameters $K_{1}^{c}=-0.7 \mathrm{eV}$ and $w=0.6 \mathrm{eV}$, appropriate to fullerides. For these values $w / 2+K_{1}^{c}<0$. As a result the band structure has nonrigid behavior with maximal enlargement in the region $2<n<4$.

This cubic model is of course too simplified to be applied directly to real systems. Nevertheless it reproduces some characteristic features of the electronic structure of alkalimetal-doped fullerides, for which orbital disproportionation of charge is expected to be a dominant feature. Indeed, it follows from Fig. 1 that the system with $n=2,4$ has an insu- lating gap of $0.8 \mathrm{eV}$ whereas in the case $n=3$ it is a metal with an enlargement of $1 \mathrm{eV}$ of the occupied bandwidth. Besides, the occupied bandwidth of the system with $n=4$ is the same as for $n=6$, but is almost twice as narrow as the occupied bandwidth in the case of $n=3$. These results are in qualitative agreement with recent photoemission data, ${ }^{13}$ where a full width at half maximum of $0.6 \mathrm{eV}$ for $\mathrm{K}_{6} \mathrm{C}_{60}$, $0.7 \mathrm{eV}$ for $\mathrm{K}_{4} \mathrm{C}_{60}$ and $1.2 \mathrm{eV}$ for $\mathrm{K}_{3} \mathrm{C}_{60}$ was observed for the occupied part of the $t_{1 u}$ LUMO band. Both $A_{2} \mathrm{C}_{60}$ and $A_{4} \mathrm{C}_{60}$ fullerides can thus be considered as band insulators with distorted noncubic band structures. In contrast correlations of the spin type are probably not strong enough in $A_{3} \mathrm{C}_{60}$ to produce a metal-insulator transition, corresponding to static one-site spin-density distortions $\Delta N_{\mathbf{m} a}^{s}, \Delta N_{\mathbf{m} \gamma_{1}}^{s}$, and $\Delta D_{\mathbf{m} \gamma_{2}}^{s} \cdot{ }^{34}$

Knupfer et al. ${ }^{11}$ have suggested that the broad band in $A_{3} \mathrm{C}_{60}$ could be accounted for within a narrow-band picture by including suitable electron-boson coupling. However, the model Hamiltonian, on which this claim was based, was oversimplified and did not contain a correct description of the interaction with fivefold degenerate phonons. In a later paper by Gunnarsson et al. ${ }^{35}$ the corrected Jahn-Teller Hamiltonian was presented in a discussion of electronphonon coupling in the photoemission spectrum of $\mathrm{C}_{60}{ }^{-}$. From this treatment it is clear that the electron-phonon interaction, accompanying the loss of one electron, is of the order of $0.1 \mathrm{eV}$, which leads mainly to single-phonon satellites in the emission spectrum, unlike the band profile observed for $A_{3} \mathrm{C}_{60}$. Furthermore, and quite surprisingly so, the authors of Ref. 11 assumed a reference half-bandwidth of $0.18 \mathrm{eV}$, i.e., almost twice as narrow as predicted by band-structure calculations. In this regime strong correlation effects are to be expected, making the one-electron band approximation in their model Hamiltonian inadequate. Finally as noted by De Seta and Evangelisti ${ }^{13}$ this model was unable to explain the room-temperature line shape of the photoemission of $\mathrm{Rb}_{3} \mathrm{C}_{60}$, which points to coupling to low-frequency modes of a different nature.

Further evidence for the orbital disproportionation in fullerides is provided by the observation of an inhomogeneous NMR spectral distribution in $\mathrm{K}_{3} \mathrm{C}_{60}$ and $\mathrm{Rb}_{3} \mathrm{C}_{60}$ at low temperatures. This points to a possible inhomogeneity of conduction-electron distribution among three inequivalent carbon atoms on each fullerene site. ${ }^{36}$ Classical bandstructure calculations are unable to reproduce sufficient population differences among the carbon atoms. ${ }^{9,37}$ In contrast, the present orbital charge disproportionation mechanism leads to relative variations of populations of carbon atoms, which are comparative with the NMR measurements. The more pronounced inhomogeneity of the NMR spectral distribution in $\mathrm{Rb}_{3} \mathrm{C}_{60}$ as compared with $\mathrm{K}_{3} \mathrm{C}_{60}$ (Ref. 36) is in agreement with enhanced disproportionation in the rubidium salt due to a narrower bandwidth.

In conclusion, we have shown that a high-symmetry distribution of conduction electrons in cubic lattices with threefold degenerate site orbitals may be subject to a special kind of band-structure instability, corresponding to orbital disproportionation of conduction-electron density at each site. This effect is caused by interelectronic interactions within the sites, and is naturally described by a set of symmetrized dis- 
proportionation components, by close analogy with symmetrized nuclear distortion coordinates in molecules. In fact the static orbital charge disproportionation and the local JahnTeller distortions of the sites will occur simultaneously and reinforce each other. In the case of fullerides we expect that the orbital charge disproportionation effect will indeed occur, with the vibronic contribution making up only $40 \%$ of the effect. Hence the electronic disproportionation mechanism is expected to become a feature of primary concern in the solid state theory of fullerides. This is already borne out by our model calculation of the effect of charge-density dis- proportionation on the nonrigidity of the $t_{1 u}$ band in a simple cubic lattice, which appears to be in semiquantitative agreement with known band features of $A_{n} \mathrm{C}_{60}$. Of course this phenomenon should be analyzed in much more detail using broken-symmetry Hartree-Fock and self-interaction corrected density-functional calculations of the band structure of alkali-metal-doped fullerides in disordered phases. Such calculations should also consider the concomitant Jahn-Teller effect of the $H_{g}$ vibrational modes in the fullerene sites.

This research was supported by a grant from the Belgian Government (Programmatie van het Wetenschapsbeleid).
*On leave from the Institute of Chemistry, Academy of Sciences, Kishinev, Moldova.

${ }^{1}$ Y. Fujishima, Y. Tokura, T. Arima, and S. Uchida, Physica C 185-189, 1001 (1989); D. A. Crandles, T. Timusk, and J. E. Greedam, Phys. Rev. B 44, 13250 (1990).

${ }^{2}$ R. C. Haddon et al., Nature (London) 350, 320 (1991); 350, 600 (1991); K. Holczer, O. Klein, S. M. Huang, R. B. Kaner, K. J. Fu, R. L. Whetten, and F. Diederich, Science 252, 1154 (1991).

${ }^{3}$ R. C. Haddon, Acc. Chem. Res. 25, 127 (1992); Physics and Chemistry of Fullerene-Based Solids-II, edited by J. E. Fisher, Y. Maruyama, D. E. Cox, and R. N. Shelton, special issue of J. Phys. Chem. Solids 54 (1993); Physics and Chemistry of Fullerenes, edited by K. Prassides (Kluwer, Dordrecht, 1994).

${ }^{4}$ S. C. Erwin, in Buckminsterfullerenes, edited by W. E. Billups and M. A. Cinfolini (VCH Publishers, New York, 1993), p. 217; S. C. Erwin and W. E. Pickett, Science 254, 842 (1991); W. Andreoni, in Properties of New Materials: Fullerenes, edited by H. Kuzmany, J. Fink, M. Mehring, and S. Roth (Springer, Berlin, 1993), Vol. 117, p. 85; S. Saito and A. Oshiyama, Phys. Rev. B 44, 11536 (1991); S. C. Erwin and M. R. Pederson, Phys. Rev. Lett. 67, 1610 (1991); J. L. Martins and N. Troullier, Phys. Rev. B 46, 1766 (1992); I. Turek and J. Hafner, ibid. 48, 14925 (1993).

${ }^{5}$ M. P. Gelfand and J. P. Lu, Phys. Rev. Lett. 68, 1050 (1992).

${ }^{6}$ S. Satpathy, V. P. Antropov, O. K. Andersen, O. Jepsen, O. Gunnarsson, and A. I. Liechtenstein, Phys. Rev. B 46, 1773 (1992).

${ }^{7}$ T. Yildirim, S. Hong, A. B. Harris, and E. J. Mele, Phys. Rev. B 48, 12262 (1993).

${ }^{8}$ S. C. Erwin and E. J. Mele, Phys. Rev. B 50, 5689 (1994).

${ }^{9}$ W. Andreoni, P. Giannozzi, and M. Parrinello, Phys. Rev. B 51, 2087 (1995).

${ }^{10}$ J. H. Weaver, Acc. Chem. Res. 25, 143 (1992); P. J. Benning, J. L. Martins, J. H. Weaver, L. P. F. Chibante, and R. E. Smalley, Science 252, 1417 (1991); J. E. Rowe, D. N. E. Buchanan, E. E. Chaban, A. F. Hebard, A. R. Kortan, A. V. Makhija, and R. C. Haddon, ibid. 252, 1419 (1991); M. Merkel, M. Knupfer, M. S. Golden, J. Fink, R. Seemann, and R. L. Johnson, Phys. Rev. B 47, 11470 (1993).

${ }^{11}$ M. Knupfer, M. Merkel, M. S. Golden, J. Fink, O. Gunnarsson, and V. P. Antropov, Phys. Rev. B 47, 13944 (1993).

${ }^{12}$ P. J. Benning, F. Stepniak, D. M. Poirier, J. L. Martins, J. H. Weaver, L. P. F. Chibante, and R. E. Smalley, Phys. Rev. B 47, 13843 (1993).

${ }^{13}$ M. De Seta and F. Evangelisti, Phys. Rev. B 51, 1096 (1995).

${ }^{14}$ M. De Seta and F. Evangelisti, Phys. Rev. Lett. 71, 2477 (1993);
R. F. Kiefl, T. L. Dut, and J. W. Schneider et al., ibid. 69, 2005 (1992).

${ }^{15}$ F. Stepniak, P. J. Benning, D. M. Poirier, and J. H. Weaver, Phys. Rev. B 48, 1899 (1993).

${ }^{16}$ M. Kosaka et al., Chem. Phys. Lett. 203, 429 (1993).

${ }^{17}$ P. W. Stephens et al., Nature (London) 351, 632 (1991); Phys. Rev. B 45, 543 (1992).

${ }^{18}$ R. M. Lof, M. A. van Veenendaal, B. Koopmans, H. T. Jonkman, and G. A. Sawatzky, Phys. Rev. Lett. 68, 3924 (1992).

${ }^{19}$ V. P. Antropov, O. Gunnarsson, and O. Jepsen, Phys. Rev. B 46, 13647 (1992).

${ }^{20}$ T. Takahashi et al., Phys. Rev. Lett. 68, 1232 (1992).

${ }^{21}$ A Fujimori et al., Phys. Rev. Lett. 69, 1796 (1992).

${ }^{22}$ D. J. Thouless, The Quantum Mechanics of Many-Body Systems (Academic, New York, 1963).

${ }^{23}$ L. M. Falikov and R. A. Harris, J. Chem. Phys. 51, 3153 (1969).

${ }^{24}$ A. Ceulemans, J. Chem. Phys. 87, 5374 (1987).

${ }^{25}$ P. W. Fowler and A. Ceulemans, Mol. Phys. 54, 767 (1985).

${ }^{26}$ In (7), $\theta$ and $\epsilon$ refer to the transformation properties of the standard functions $d_{z^{2}}$ and $d_{x^{2}-y^{2}}$, respectively. Note that a different convention, which is more appropriate for icosahedral groups is used in Ref. 25.

${ }^{27}$ I. B. Bersuker and V. Z. Polinger, Vibronic Interactions in Molecules and Crystals (Springer-Verlag, Berlin, 1989), p. 81.

${ }^{28}$ R. Parr and W. Yang, Density-Functional Theory of Atoms and Molecules (Oxford University Press, New York, 1989), p. 180.

${ }^{29}$ N. Koga and K. Morokuma, Chem. Phys. Lett. 196, 191 (1992).

${ }^{30}$ N. Manini, E. Tosatti, and A. Auerbach, Phys. Rev. B 49, 13008 (1994).

${ }^{31} \mathrm{O}$. Madelung, Introduction to Solid-State Theory (SpringerVerlag, Berlin, 1978), p. 340.

${ }^{32}$ Note that Mott-Hubbard transition requires an integer number of electrons per site, $n$, which was not supposed in our calculations.

${ }^{33}$ R. M. Fleming et al., Nature (London) 352, 701 (1991); A. Lappas, M. Kosaka, K. Tanigaki, and K. Prassides, J. Am. Chem. Soc. 117, 7560 (1995); Z. Gamba and G. Martinez, J. Chem. Phys. 102, 8132 (1995).

${ }^{34}$ It was pointed out, however, that the fullerides $A_{3} \mathrm{C}_{60}$ are near the edge of the Mott-Hubbard transition (Refs. 10, 12, 13, and 19), therefore significant dynamic spin correlations can be expected.

${ }^{35}$ O. Gunnarsson, H. Handschuh, P. S. Bechthold, B. Kessler, G. Ganteför, and W. Eberhardt, Phys. Rev. Lett. 74, 1875 (1995).

${ }^{36}$ K. Holczer et al., Europhys. Lett. 23, 63 (1993).

${ }^{37}$ V. P. Antropov, I. I. Mazin, O. K. Andersen, A. I. Liechtenstein, and O. Jepsen, Phys. Rev. B 47, 12373 (1993). 